PROCEEDINGS OF THE

AMERICAN MATHEMATICAL SOCIETY

Volume 130, Number 8, Pages 2471-2477

S 0002-9939(02)06406-7

Article electronically published on January 23, 2002

\title{
COMMUTATOR SUBGROUPS AND FOLIATIONS WITHOUT HOLONOMY
}

\author{
TAO LI
}

(Communicated by Ronald A. Fintushel)

\begin{abstract}
Suppose a manifold $M$ has a codimension one, transversely orientable foliation without holonomy, and $L$ is a leaf. We give a simple, purely topological proof of the theorem that $\pi_{1}(L)$ is a normal subgroup containing the commutator subgroup of $\pi_{1}(M)$.
\end{abstract}

\section{INTRODUCTION}

Let $M$ be a compact $n$-dimensional manifold. If $M$ is a fiber bundle over $S^{1}$ with fiber a compact $n-1$ dimensional manifold $S$, then $\pi_{1}(S)$ is a normal subgroup of $\pi_{1}(M)$ and the quotient group $\pi_{1}(M) / \pi_{1}(S)$ is isomorphic to $\mathbb{Z}$. Thus, the commutator subgroup of $\pi_{1}(M)$ is a subgroup of $\pi_{1}(S)$. This simple fact can be generalized to codimension one foliations without holonomy. We say that a foliation has no holonomy if the holonomy group of every leaf is trivial. A fiber bundle is an obvious example of foliation without holonomy. Moreover, if a foliation is defined by a closed non-singular one form, then it has no holonomy by Reeb [7], and the converse is also true for foliations of class $C^{2}$ by Sacksteder [10].

Theorem 1.1. Let $M$ be a compact manifold and $\mathcal{F}$ be a codimension one transversely orientable $C^{0}$ foliation of $M$. Suppose that $\mathcal{F}$ has no holonomy in the interior of $M$. Then, for any leaf $L$ of $\mathcal{F}$ that is not a component of $\partial M, i_{*}\left(\pi_{1}(L)\right)$ is a normal subgroup of $\pi_{1}(M)$ containing the commutator subgroup of $\pi_{1}(M)$, where $i_{*}: \pi_{1}(L) \rightarrow \pi_{1}(M)$ is the homomorphism induced by the inclusion map $i$.

If we assume $\mathcal{F}$ to be a foliation of class $C^{2}$, then Theorem 1.1 follows from a powerful theorem of Sacksteder [10]. In Theorem 1.1, if we assume that there exists a flow on $M$ transverse to $\mathcal{F}$, then Theorem 1.1 can be proved using Imanishi's techniques plus Hölder's theorem on archimedian-ordered groups [4, 6]. In the proof of the theorem of Sacksteder [10], Hölder's theorem also plays an important role. However, Hölder's theorem is a purely algebraic theorem, and it would be more interesting to have a proof of Theorem 1.1 that depends only on topological properties of the foliation. In this paper, we prove Theorem 1.1 without the assumptions on differentiability of the foliation or existence of a transverse flow, and the proof is a simple topological argument that does not involve Hölder's theorem. Note that the assumption on existence of a transverse flow is not essential in Imanishi's techniques.

Received by the editors October 2, 2000 and, in revised form, March 9, 2001.

2000 Mathematics Subject Classification. Primary 57N10, 57R30.

(C)2002 American Mathematical Society 
Corollary 1.2 (Rosenberg). Let $M$ be a closed 3-manifold with a foliation each of whose leaves is homeomorphic to $\mathbb{R}^{2}$. Then $\pi_{1}(M)$ is free abelian, and hence $M$ is homeomorphic to $S^{1} \times S^{1} \times S^{1}$.

Rosenberg has proved Corollary 1.2 for $C^{2}$ foliations 88 , and it is well known to be true for $C^{0}$ foliations [3].

The motivation for this paper is to find a direct topological proof for Corollary 1.2, and this topological proof is a small step in the author's program of finding an algorithm to decide whether a 3-manifold contains essential laminations. It has been shown in 5 that a branched surface with certain combinatorial conditions always carries an essential lamination, and an essential lamination is always carried by such a branched surface unless it is a lamination by planes. Since laminations (and foliations) by planes are the only exceptional cases, a topological proof for Corollary 1.2 may help to gain insight into the decision problem about existence of essential laminations in 3-manifolds.

\section{Proof of Theorem 1.1}

We can assume that $M$ is an $n$-dimensional manifold with $n \geq 3$, since the case of $n \leq 2$ is trivial. By "blowing air" into leaves, i.e., replacing leaves in $\mathcal{F}$ by $I$-bundles over these leaves and deleting the interior of these $I$-bundles, we can deform the foliation into a lamination, which we denote by $\lambda$. This well-known operation is essentially due to Denjoy [2], and if Theorem [1.1] holds for the leaves in the lamination $\lambda$, it holds for the foliation $\mathcal{F}$. Moreover, we can assume that $\lambda$ lies in the interior of a compact subset $N(B)$ of $M$. Here, $N(B)$ is a union of finitely many embedded $D^{n-1} \times I^{\prime}$ 's $\left(D^{n-1}\right.$ is an $(n-1)$-dimensional disk), and the intersection of any two $D^{n-1} \times I$ 's is either empty, or an $(n-1$ dimensional) disk in their vertical boundaries (i.e. $\partial D^{n-1} \times I$ 's) consisting of a union of subarcs of the $I$-fibers in $\partial D^{n-1} \times I$. We can construct $N(B)$ by considering the foliation charts of $\mathcal{F}$. After we "blow air" into leaves, each foliation chart can be deformed into finitely many $D^{n-1} \times I$ 's, and these $D^{n-1} \times I$ 's can be glued together to form $N(B)$. Furthermore, by splitting $N(B)$ a little, we can assume that only finitely many $I$-fibers in these $\partial D^{n-1} \times I$ 's can be glued together to form an $I$-fiber in $N(B)$. So, $N(B)$ can be considered as a union of $I$-fibers whose endpoints lie in $\partial N(B)$, and $\lambda$ is transverse to each $I$-fiber of $N(B)$. If we collapse each $I$-fiber in $N(B)$ to a point, $N(B)$ becomes an $(n-1)$-dimensional complex $B$, which is an analogue to branched surfaces in 3-manifolds. Since $\mathcal{F}$ is transversely orientable, $\lambda$ is transversely orientable, and we can give each $I$-fiber of $N(B)$ a direction induced from the transverse orientation of $\mathcal{F}$ and $\lambda$.

Definition 2.1. We say a curve $l$ in $M$ is transverse to $N(B)$ if its intersection with $N(B)$ is a union of subarcs of the $I$-fibers in $N(B)$, and there exists a direction along $l$ that is compatible with the direction of each arc in $l \cap N(B)$ (induced from the transverse orientation of $\mathcal{F}$ ). We call a curve $l$ in $M$ piecewise transverse to $N(B)$, if $l$ can be decomposed into a union of finitely many $\operatorname{arcs} a_{i}$ and $b_{i}$ with $\partial a_{i}=x_{2 i-1} \cup x_{2 i}$ and $\partial b_{i}=x_{2 i} \cup x_{2 i+1}$, such that:

(1) each $b_{i}$ is transverse to $N(B)$;

(2) each $a_{i}$ lies in a leaf of $\lambda$;

(3) there exists a direction along $l$ that is compatible with the direction of each $b_{i}$ (induced from the transverse orientation of $\mathcal{F}$ ). 
A normal fence is a continuous map $H:[0,1] \times J \rightarrow M$, where $J$ is an interval, such that $H([0,1] \times\{s\})$ either lies in a leaf of $\lambda$ or lies in $M-\lambda$ (for any $s \in J$ ), and $\left.H\right|_{\{t\} \times J}$ is a curve transverse to $N(B)$ for each $t$. We call $H:[0,1] \times J \rightarrow M$ a trivial fence if the image of $H$ (i.e. $H([0,1] \times J)$ ) lies in a single leaf of $\lambda$. We call $H:[0,1] \times J \rightarrow M$ a piecewise normal fence, if there exist $t_{0}=0<t_{1}<\cdots<t_{k}=1$ such that each $\left.H\right|_{\left[t_{i}, t_{i+1}\right] \times J}$ is either a trivial fence or a normal fence. We say that a curve or a surface intersects $\lambda$ transversely if it is transverse to the leaves of $\lambda$.

The following proposition is an obvious fact.

Proposition 2.2. Let $\eta_{1}$ and $\eta_{2}$ be two arcs piecewise transverse to $N(B)$, and let $\alpha$ be an arc in a leaf of $\lambda$ with two endpoints lying in the interior of $\eta_{1}$ and $\eta_{2}$ respectively. Suppose each component of $\eta_{i}-\partial \alpha$ contains a subarc of an I-fiber of $N(B)$. Then, there is a piecewise normal fence $H:[0,1] \times(-\delta, \delta) \rightarrow M$ such that $H([0,1] \times\{0\})=\alpha$, and $\left.H\right|_{[0,1] \times(\delta-\epsilon, \delta)}$ and $\left.H\right|_{[0,1] \times(-\delta,-\delta+\epsilon)}$ are normal fences for some small $\epsilon$.

Proof. If both $\eta_{1}$ and $\eta_{2}$ are transverse to $N(B)$, then the proof is trivial. Otherwise, by gluing trivial fences and normal fences together, we can easily construct such a map.

Lemma 2.3 is due to Imanishi [4], and the proof is a simple topological argument; see also [1. Imanishi's proof is for foliations, but it is easy to see that his argument works as well for laminations using our definition of normal fence.

Lemma 2.3 (Imanishi 4]). Let $M$ and $\lambda$ be as above. Then every normal fence $H:[0,1] \times[a, b) \rightarrow M$ can be extended continuously to $H:[0,1] \times[a, b] \rightarrow M$, if $H(\{0\} \times[a, b))$ can be extended continuously to $H(\{0\} \times[a, b])$ with $H(0, b) \in \lambda$.

Since $\mathcal{F}$ has no holonomy in $M-\partial M$, each leaf of $\mathcal{F}$ that is not in $\partial M$ must be $\pi_{1}$-injective by Novikov [6], and hence each leaf $L$ in $\lambda$ is $\pi_{1}$-injective. So, to simplify notation, we do not distinguish $i_{*}\left(\pi_{1}(L)\right)$ and $\pi_{1}(L)$, where $L$ is a leaf in $\lambda$.

Lemma 2.4. Let $M$ and $\lambda$ be as above, let $\gamma$ be a closed curve in the interior of $M$, let $x$ be a point in $\gamma \cap \lambda$, and let $L_{x}$ be the leaf of $\lambda$ that contains $x$. Then either $\gamma$ represents an element in $\pi_{1}\left(L_{x}, x\right)$, or $\gamma$ can be homotoped to be piecewise transverse to $N(B)$ by a homotopy fixing $x$. Moreover, if $\gamma$ does not represent an element in $\pi_{1}\left(L_{x}, x\right)$, after some further homotopy, $\gamma$ intersects $\lambda$ transversely, and $\gamma$ admits a direction (along $\gamma$ ) that is compatible with the transverse orientation of $\lambda$.

Proof. If we can homotope $\gamma$ to be piecewise transverse to $N(B)$, then by pinching each subarc of $\gamma$ that lies in a leaf to a point, we can easily homotope $\gamma$ to be transverse to $\lambda$. By our definition for arcs piecewise transverse to $N(B)$, after this homotopy, $\gamma$ admits a direction (along $\gamma$ ) that is compatible with the transverse orientation of $\lambda$.

Suppose that $\gamma$ is not piecewise transverse to $N(B)$, and does not lie in $L_{x}$. Then, after some homotopy fixing $x$, we can assume that $\gamma$ is decomposed into a union of $2 n \operatorname{arcs} a_{i}$ and $b_{i}(i=1, \ldots, n)$ such that $a_{i}$ and $b_{i}$ alternately appear on $\gamma$, each $a_{i}$ lies in a leaf of $\lambda$, each $b_{i}$ is piecewise transverse to $N(B)$, and each endpoint of $b_{i}$ has a small neighborhood in $b_{i}$ that is a subarc of an $I$-fiber of $N(B)$. Each $b_{i}$ has a direction (as in the definition) compatible with the transverse orientation 
of $\mathcal{F}$. Suppose that the boundary points of these arcs are $x_{1}, x_{2}, \ldots, x_{2 n}$, where $\partial a_{i}=x_{2 i-1} \cup x_{2 i}$ and $\partial b_{i}=x_{2 i} \cup x_{2 i+1}\left(x_{2 n+1}=x_{1}\right)$.

Now, we consider the two arcs $b_{i-1}$ and $b_{i}$ adjacent to $a_{i}\left(b_{0}=b_{2 n}\right)$. If the direction of $b_{i-1}$ points towards $x_{2 i-1}$ and the direction of $b_{i}$ points away from $x_{2 i}$, then $b_{i-1} \cup a_{i} \cup b_{i}$ is piecewise transverse to $N(B)$ and the number of those arcs (i.e. $2 n$ ) is reduced. Therefore, we can assume that directions of $b_{i-1}$ and $b_{i}$ either both point towards $a_{i}$, or both point away from $a_{i}$.

By the argument above, $n$ must be greater than one, since, if $n=1$, the direction of $b_{1}$ points towards one endpoint of $a_{1}$ and away from the other endpoint, and hence $\gamma$ is piecewise transverse to $N(B)$. As $n>1$, we can assume that $x \notin a_{2}$. By Proposition 2.2 and our assumption about the directions of $b_{i}$ 's, there is a piecewise normal fence $H:[1,2] \times[0, \delta) \rightarrow M$ such that $H([1,2] \times\{0\})=a_{2}$ and $\left.H\right|_{\{i\} \times[0, \delta)}$ is an arc in $b_{i}(i=1,2)$. The normal fence can be continuously extended to $H:[1,2] \times[0, \delta] \rightarrow M$ by Lemma 2.3 We assume $H$ to be maximal, i.e. there is no normal fence $H^{\prime}:[1,2] \times[0, t) \rightarrow M$ such that $\left.H^{\prime}\right|_{\{i\} \times[0, t)}$ is an arc in $b_{i}(i=1,2)$ and $H=\left.H^{\prime}\right|_{[1,2] \times[0, \delta]}$ where $\delta<t$. If $H(i, \delta)$ lies in the interior of $b_{i}$ for both $i=1,2$, then by Proposition 2.2 there is a piecewise normal fence $K:[1,2] \times[0, \epsilon) \rightarrow M$ such that $\left.K\right|_{[1,2] \times\{0\}}=\left.H\right|_{[1,2] \times\{\delta\}}$ and $\left.K\right|_{\{i\} \times[0, \epsilon)}$ is an arc in $b_{i}-H(\{i\} \times[0, \delta])$ $(i=1,2)$. Then, we can glue $H$ and $K$ together to form a larger piecewise normal fence, i.e., we can construct a piecewise normal fence $H^{\prime}:[1,2] \times[0, \delta+\epsilon) \rightarrow M$, where $H^{\prime}(y, s)=H(y, s)$ if $s \in[0, \delta]$ and $H^{\prime}(y, s)=K(y, s-\delta)$ if $s \in[\delta, \delta+\epsilon)$. This contradicts our assumption that $H$ is maximal. Thus, at least one of $H(1, \delta)$ and $H(2, \delta)$ is an endpoint of $b_{1} \cup b_{2}$.

Suppose $H(2, \delta) \in \partial b_{2}$. Then $H(2, \delta)$ must be $x_{5}$ and $H(\{2\} \times[0, \delta])=b_{2}$. If $x \notin H(\{1\} \times(0, \delta)) \cup\left(b_{2}-\partial b_{2}\right)$, then we can homotope $H(\{1\} \times[0, \delta]) \cup a_{2} \cup b_{2}$ to $H([1,2] \times\{\delta\})$ fixing the remaining part of $\gamma$. This homotopy fixes $x$ (since $x \notin a_{2}$ ) and reduces the number $n$. Note that if $x$ is one of the $x_{i}$ 's (other than $\partial a_{2}$ ), the number $n$ is reduced after this process. By assuming $n$ to be minimal, we have that $x$ lies in $H(\{1\} \times(0, \delta)) \cup b_{2}$ (since $\left.x \notin a_{2}\right)$, and $x$ is an endpoint of $H([1,2] \times\{t\}$ ) for some $t \in(0, \delta)$. Next, we homotope $H(\{1\} \times[0, t]) \cup a_{2} \cup H(\{2\} \times[0, t])$ to $H([1,2] \times\{t\})$ and fix the remaining part of $\gamma$. Let $H([1,2] \times\{t\})$ be $a_{2}^{\prime}$, let $b_{i}-$ $H(\{i\} \times[0, t))$ be $b_{i}^{\prime}(i=1,2)$, and let the whole of the closed curve after this homotopy be $\gamma^{\prime}$. Then $a_{2}^{\prime}$ lies in a leaf, $b_{i}^{\prime}$ is piecewise transverse to $N(B)(i=1,2)$, $x$ is an endpoint of $a_{2}^{\prime}$, and $\gamma^{\prime}$ is a union of $2 n$ such arcs. Since $x$ is an endpoints of $a_{2}^{\prime}$, after we apply the same process to $\gamma^{\prime}$ (using $a_{1}$ to construct the map $H$ ), the number $n$ is reduced. Therefore, in any case, we can reduce the number $n$ by homotopies fixing $x$, and the lemma follows.

Corollary 2.5. Let $M$ and $\lambda$ be as above. Then, for any leaf $L \subset \lambda$ that is not a component of $\partial M, \pi_{1}(L)$ is a normal subgroup of $\pi_{1}(M)$.

Proof. We need to show that, for any $g \in \pi_{1}(L, x)$ and $f \in \pi_{1}(M, x)-\pi_{1}(L, x)$, $f^{-1} \circ g \circ f \in \pi_{1}(L, x)$, where $x \in L$. Since $f \notin \pi_{1}(L, x)$, by Lemma 2.4, $f$ can be represented by a closed curve, which we denote by $\beta$, piecewise transverse to $N(B)$. Let $\alpha$ be a curve in $L$ that represents $g$, and $x=\alpha \cap \beta$. By Proposition 2.2 and Lemma 2.4, there is a piecewise normal fence $H:[0,1] \times[0, \delta] \rightarrow M$ such that $H([0,1] \times\{0\})=\alpha, H(i, 0)=x$ and $\left.H\right|_{\{i\} \times[0, \delta]}$ is an arc in $\beta(i=0,1)$. By choosing $H$ to be maximal, as in the proof of Lemma [2.4 we can assume that at least one of $H(0, \delta)$ and $H(1, \delta)$ is $x$. 


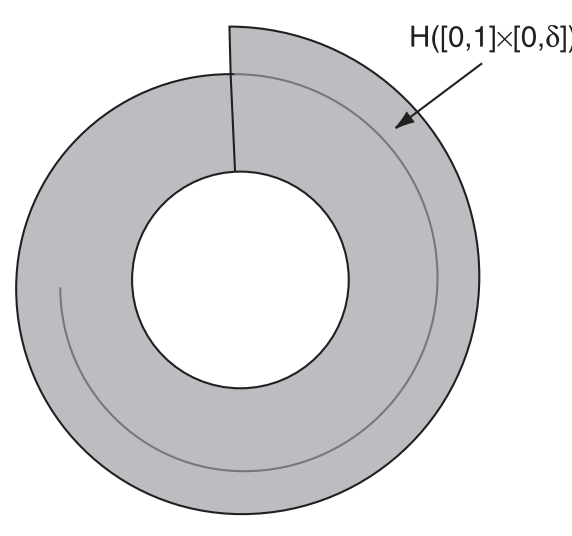

(a)

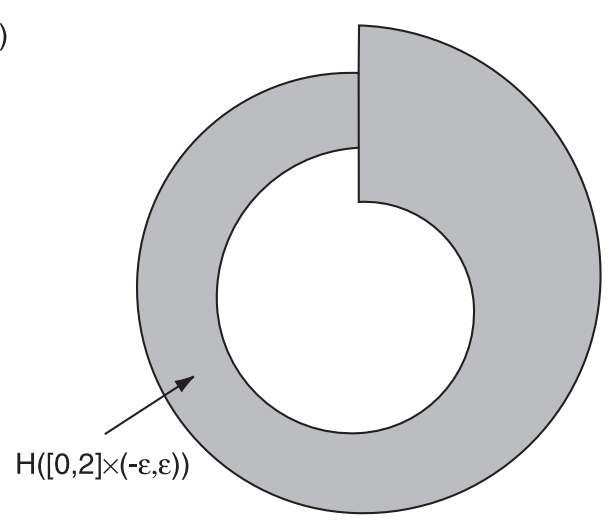

(b)

FIGURE 1.

If $H(0, \delta)=H(1, \delta)=x$, then $H(\{0\} \times[0, \delta])=H(\{1\} \times[0, \delta])=\beta$ and $H(\{0\} \times$ $[0, \delta]) \cup H([0,1] \times\{0\}) \cup H(\{1\} \times[0, \delta])$ can be homotoped to $H([0,1] \times\{\delta\}) \subset L$ fixing $H(i, \delta)=x(i=0,1)$. Hence, the curve $H([0,1] \times\{\delta\}) \subset L$ represents $f^{-1} \circ g \circ f$, i.e. $f^{-1} \circ g \circ f \in \pi_{1}(L, x)$.

Suppose that $H(0, \delta)=x$ and $H(1, \delta) \neq x$. Then $H(\{1\} \times[0, \delta])$ must be a subarc in $H(\{0\} \times[0, \delta))$, as shown in Figure 1 (a). After some small homotopy on $H$, we can assume that $H$ intersects $\lambda$ transversely, $H^{-1}(\lambda)$ is a union of compact arcs connecting $\{0\} \times[0, \delta]$ to $\{1\} \times[0, \delta]$, and $H(\{1\} \times[0, \delta])$ is glued to a subarc in $H(\{0\} \times[0, \delta))$ as shown in Figure 1 (a). In Figure 1 (a), the curve comes into the shaded region, i.e. the leaf in $H^{-1}(\lambda)$ containing $H([0,1] \times\{\delta\})$ must converge to a limit cycle as in the Poincaré-Bendixson theorem, which gives a curve with nontrivial holonomy. This contradicts our hypothesis that $\mathcal{F}$ (and hence $\lambda$ ) has no holonomy in $M-\partial M$.

Corollary 2.6. Let $M$ and $\lambda$ be as above, and let $l$ be a closed curve in $M-\partial M$ that is transverse to $\lambda$ and admits a direction along $l$ compatible with the transverse orientation of $\lambda$. Then l has non-empty intersection with every leaf in $M-\partial M$.

Proof. Suppose that there is a leaf $L$ in $M-\partial M$ whose intersection with $l$ is empty. Then there is another curve $l^{\prime}$ that is homotopic to $l$ but has non-empty intersection with $L$. Let $x \in l^{\prime} \cap L$. By Lemma 2.4, $l^{\prime}$ can be homotoped (fixing $x$ ) to a curve $l^{\prime \prime}$ that either lies inside $L$, or transversely intersects $\lambda$ with a compatible direction along $l^{\prime \prime}$. Since $l$ and $l^{\prime \prime}$ are homotopic, there is a map $P: S^{1} \times[0,1] \rightarrow M-\partial M$ such that $P\left(S^{1} \times\{0\}\right)=l$ and $P\left(S^{1} \times\{1\}\right)=l^{\prime \prime}$.

Suppose $l^{\prime \prime} \subset L$. Then, after some homotopy, we can assume that $P\left(S^{1} \times[0,1)\right)$ intersects $\lambda$ transversely. Since $l$ admits a direction compatible with the transverse orientation of $\mathcal{F}, P^{-1}(\lambda)$ contains no compact arcs with both end points in $S^{1} \times\{0\}$. Since $l \cap L=\emptyset, P^{-1}(\lambda)$ contains no compact arcs connecting $S^{1} \times\{0\}$ to $S^{1} \times\{1\}$. As in the Poincaré-Bendixson theorem, every curve in $P^{-1}(\lambda)$ that intersects $l=$ $S^{1} \times\{0\}$ must converge to a limit cycle, which contradicts our hypothesis that $\mathcal{F}$ (and hence $\lambda$ ) has no holonomy. 
So, we can assume that $l^{\prime \prime}$ transversely intersects $\lambda$ with a compatible direction along $l^{\prime \prime}$. After some homotopy, we can assume that $P\left(S^{1} \times[0,1]\right)$ intersects $\lambda$ transversely and $P^{-1}(\lambda)$ is a one-dimensional lamination in $S^{1} \times[0,1]$. Then, there is no compact arc in $P^{-1}(\lambda)$ with both endpoints in the same boundary circle $S^{1} \times\{i\}(i=0$ or 1$)$. Let $y \in S^{1} \times\{1\}$ such that $P(y)=x \in L$, and we denote the one-dimensional leaf in $P^{-1}(\lambda)$ containing $y$ by $l_{y}$. Since $l \cap L=\emptyset, l_{y} \cap S^{1} \times\{0\}=\emptyset$ and $l_{y}$ must converge to a limit cycle as in the Poincaré-Bendixson theorem, which gives a curve in $\lambda$ with nontrivial holonomy. This also gives a contradiction.

Next, we show that, for any leaf $L$ in $\lambda$ that is not a component of $\partial M$, the commutator subgroup of $\pi_{1}(M)$ is a subgroup of $\pi_{1}(L)$. It suffices to show that each commutator in $\pi_{1}(M, x)$ must lie in $\pi_{1}(L, x)(x \in L)$. Let $\eta$ be a curve that represents a commutator $c$ of $\pi_{1}(M, x)$. Suppose that $c \notin \pi_{1}(L, x)$. Then, by Lemma 2.4 we can assume that $\eta$ transversely intersects $\lambda$ and admits a direction along $\eta$ that is compatible with the transverse orientation of $\lambda$. Since $c$ is a commutator, there is a map $f: T \rightarrow M$ such that $f(\partial T)=\eta$, where $T$ is a once-punctured torus. After some homotopy, we can assume that $f(T)$ intersects $\lambda$ transversely, and hence $f^{-1}(\lambda)$ is a one-dimensional lamination in $T$.

Since $\eta$ admits a direction along $\eta$ that is compatible with the transverse orientation of $\lambda$, there is no leaf in $f^{-1}(\lambda)$ that is a compact arc with both endpoints in $\partial T$. Thus, any leaf in $f^{-1}(\lambda)$ that meets $\partial T$ must be a ray with one boundary point in $\partial T$ and one end. Let $r$ be such a ray. Then the closure of $r$, which we denote by $\bar{r}$, is a sub-lamination of $f^{-1}(\lambda)$. If there is a circular leaf in $\bar{r}$, then it must have non-trivial holonomy, which gives a contradiction.

Before we proceed, we show that a ray cannot lie in the limit of a ray or a line (a line a leaf in $f^{-1}(\lambda)$ that is homeomorphic to $\mathbb{R}$ ). Suppose there is a ray $r^{\prime}$ lying in the limit of $\rho$, where $\rho$ is either a ray or a line in $f^{-1}(\lambda)$. Then, the endpoint of $r^{\prime}$ is the limit of a series of points in $\rho$. In other words, near the endpoint of $r^{\prime}$, there is an embedding $h:[0,1] \times(-\epsilon, \epsilon) \rightarrow T$ such that $h(\{1\} \times(-\epsilon, \epsilon)) \subset \partial T$, $h([0,1] \times\{0\}) \subset r^{\prime}$, and $h^{-1}(\rho)$ is a union of arcs of the form $[0,1] \times\{t\}(t \in(-\epsilon, \epsilon))$. This implies that $\rho$ intersects $\partial T$ infinitely many times, which is absurd. So, $\bar{r}$ cannot be a union of rays, and hence $\bar{r}$ must contain a line.

Let $\mu$ be the union of lines in $f^{-1}(\lambda)$. By the argument above, $\mu$ is not empty and the closure of $\mu$ contains neither circles nor rays. Hence, $\mu$ is a closed set. Let $C$ be the component of $T-\mu$ that contains $\partial T$. Since $T$ is a punctured torus, topologically, $C$ is a region of $(0,1) \times \mathbb{R}$ with an open disk removed. $C$ has two infinitesimal ends, and the ends of the rays in $f^{-1}(\lambda)$ must lie in the same infinitesimal end of $C$, since $\eta=\partial T$ admits a direction along $\eta$ that is compatible with the transverse orientation of $\lambda$. Therefore, by considering $C$ as a product region of $(0,1) \times \mathbb{R}$ with an open disk removed, we can assume that there is an embedding $j:(0,1) \times[0,+\infty) \rightarrow C$, whose image is correspondent to an infinitesimal end of $C$, such that $r-j((0,1) \times(0,+\infty))$ is compact for any ray $r$ in $f^{-1}(\lambda)$. Let $R_{1}$ and $R_{2}$ be the two lines in $\mu$ that bound $C$, and $z$ be a limit point of $R_{1} \cup R_{2}$. Then, for any open $\operatorname{arc} J$ in $T$ that contains $z$ and transversely intersects $f^{-1}(\lambda)$, the infinitesimal ends of $C$ must intersect $J$ infinitely many times, i.e., $J \cap j((0,1) \times(0,+\infty))$ must have infinitely many components. In particular, we can assume that there is a number $N_{J}$ such that $j\left((0,1) \times\left\{N_{J}\right\}\right) \subset J$.

By Corollary [2.6, the leaf of $\lambda$ that contains $f(z)$ must have non-empty intersection with $\eta$. Hence, there is an embedding $H_{0}:[0,1] \times(-\epsilon, \epsilon) \rightarrow M$ such 
that: $H_{0}(\{s\} \times(-\epsilon, \epsilon))$ is transverse to $\lambda$ for any $s \in[0,1], H_{0}(0,0)=f(z)$, $H_{0}(\{0\} \times(-\epsilon, \epsilon)) \subset f(T), H_{0}(\{1\} \times(-\epsilon, \epsilon)) \subset \eta$, and each leaf in the lamination $H_{0}^{-1}(\lambda)$ is of the form $[0,1] \times\{t\}$ for some $t \in(-\epsilon, \epsilon)$. Let $J$ be the component of $f^{-1}\left(H_{0}(\{0\} \times(-\epsilon, \epsilon))\right)$ that contains $z$; then we can assume $J$ to be an open interval in $T$ transverse to $f^{-1}(\lambda)$. By our argument above, there is an embedding $k:[1,2] \times(-\epsilon, \epsilon) \rightarrow C$ such that $k(\{1\} \times(-\epsilon, \epsilon))=f^{-1}\left(H_{0}(\{1\} \times(-\epsilon, \epsilon))\right) \subset \partial T$, $k(\{2\} \times(-\epsilon, \epsilon)) \subset J$, and the leaves of the lamination $k^{-1}\left(f^{-1}(\lambda)\right)$ is a union of parallel arcs connecting $\{1\} \times(-\epsilon, \epsilon)$ to $\{2\} \times(-\epsilon, \epsilon)$. Let $H$ be the concatenation of $H_{0}$ and $k$, i.e. $H:[0,2] \times(-\epsilon, \epsilon) \rightarrow M$ be the map that $H(p, q)=H_{0}(p, q)$ if $p \in[0,1]$ and $H(p, q)=f \circ k(p, q)$ if $p \in[1,2]$. Therefore, $H^{-1}(\lambda)$ is a union of parallel arcs connecting $\{0\} \times(-\epsilon, \epsilon)$ to $\{2\} \times(-\epsilon, \epsilon)$, and $H(\{2\} \times(-\epsilon, \epsilon))$ is glued to a proper open arc in $H(\{0\} \times(-\epsilon, \epsilon))$, as shown in Figure 1 (b). Then, similar to our argument for Figure 1 (a) and the Poincaré-Bendixson theorem, there must be a closed curve in $\lambda$ with non-trivial holonomy. This contradicts our hypothesis that $\mathcal{F}$ has no holonomy in $M-\partial M$, and Theorem 1.1 follows.

\section{REFERENCES}

[1] A. Candel and L. Conlon, Foliations. I. Graduate Studies in Mathematics, 23, Amer. Math. Soc., Providence, RI, 2000, pp. 208-211. CMP 2000:06

[2] A. Denjoy, Sur les courbes définies par les équations différentielles à la surface du tore, J. Math. Pures Appl. 11 (1932), 333-375.

[3] D. Gabai, Foliations and 3-manifolds, Proceedings of the International Congress of Mathematicians, Vol. I, II (Kyoto, 1990), 609-619, Math. Soc. Japan, Tokyo, 1991. MR 93d:57013

[4] H. Imanishi, On the theorem of Denjoy-Sacksteder for codimension one foliations without holonomy. J. Math. Kyoto Univ. 14 (1974), 607-634. MR 51:4270

[5] T. Li, Laminar branched surfaces in 3-manifolds, Preprint.

[6] S. Novikov, Topology of foliations, Trans. Moscow Math. Soc. 14 (1965), 268-305. MR 34:824

[7] G. Reeb, Sur la courbure moyenne des variétés intégrales d'une équation de Pfaff $\omega=0$. C. R. Acad. Sci. Paris 231 (1950). 101-102. MR 12:54c

[8] H. Rosenberg, Actions of $\mathbb{R}^{n}$ on manifolds. Comment. Math. Helv. 41 (1966), 170-178. MR 34:6794

[9] H. Rosenberg, Foliations by planes. Topology, 7 (1968) 131-138. MR 37:3595

[10] R. Sacksteder, Foliations and pseudo-groups, Amer. J. Math. 87 (1965) 79-102. MR 30:4268

Department of Mathematics, C1200, University of Texas at Austin, Austin, Texas 78712

E-mail address: taoli@math.utexas.edu

Current address: Department of Mathematics, 401 Mathematical Sciences, Oklahoma State University, Stillwater, Oklahoma 74078

E-mail address: tli@math.okstate.edu 